EXTENDED REPORT

\title{
Quality of life and visual function in patients with intermediate uveitis
}

\author{
C C Murphy, E H Hughes, N A Frost, A D Dick
}

Br J Ophthalmol 2005;89:1161-1165. doi: 10.1136/bjo.2005.067421

See end of article for authors' affiliations

......................

Correspondence to: Professor Andrew Dick Department of Clinical Sciences, University of Bristol, Bristol Eye Hospital, Lower Maudlin Street, Bristol BS1 2LX, UK; a.dick@bristol.ac.uk

Accepted for publication 1 March 2005

\begin{abstract}
Aims: To assess visual function, vision related quality of life (VR-QOL), and general health related quality of life (HR-QOL) in intermediate uveitis (IU).

Methods: VR-QOL and HR-QOL were evaluated in 42 patients with IU using the VCMI and SF-36 questionnaires, respectively. LogMAR visual acuity (VA), Pelli-Robson contrast sensitivity (CS), FarnsworthMunsell 100 hue colour vision (CV), and Estermann visual field (VF) were recorded monocularly and binocularly.

Results: Median (interquartile range) visual acuity (VA) and CS of 72 affected eyes were 0.1 (0.015-0.3) and 1.55 (1.35-1.65), respectively. 9.5\% of patients had a VCM1 score of more than 2.0, indicating "more than a little" concern over vision. Worse eye VA $(p=0.045)$ and CS $(p=0.042)$ were predictive of a VCM1 score of more than 2.0 independently of age, sex, uveitis duration, laterality and activity, systemic uveitis therapy, and medical co-morbidity. The physical and mental component summary scores of the SF36 were significantly worse in those who reported significant impairment of vision on the VCMI than those who did not.
\end{abstract}

Conclusions: The majority of patients with IU maintain good visual function and quality of life. VR-QOL impairment in IU correlates with vision in the worse eye and is associated with impaired HR-QOL.
1 ntermediate uveitis is a type of chronic relapsing uveitis characterised by vitritis, peripheral retinal vasculitis, cystoid macular oedema, and the absence of significant chorioretinal inflammation. Its impact on visual function and quality of life is not accurately known.

Visual acuity is usually the only measure of visual function routinely tested in uveitis clinics. ${ }^{1}$ However, considered alone it inadequately describes visual performance, which can be affected by other measures of visual function including colour vision, contrast sensitivity, and visual field. It is becoming increasingly recognised that vision related quality of life (VR-QOL) questionnaires, which measure the global impact of visual impairment on physical, psychological, and social functioning in day to day life, provide an additional and effective means of measuring visual functioning, as has been demonstrated for patients with cataract, glaucoma, macular disease and, more recently, uveitis. ${ }^{2-6}$ In addition to using VR-QOL instruments to measure the effect of visual impairment on quality of life, generic instruments can be used to measure overall health related quality of life (HRQOL), such as the short form 36 (SF-36) questionnaire. ${ }^{7}$

In this study we performed a comprehensive evaluation of the impact of intermediate uveitis on visual functioning by measuring visual acuity, contrast sensitivity, colour vision, and visual field. We explored patients' perceptions of their vision and health related quality of life and attempted to identify what clinical and demographic factors contributed to their impaired health status. We elected to study intermediate uveitis in this context because it is a clearly defined clinical entity, has a relatively small number of causes, and is more common than most sight threatening uveitis entities.

\section{PATIENTS AND METHODS}

\section{Patient group}

In all, 42 consecutive patients with intermediate uveitis attending a specialist uveitis clinic were enrolled in this study between August and December 2002. Two patients who were asked to participate during the study period declined involvement. The diagnosis of intermediate uveitis was based on the presence of vitreous inflammation, inferior vitreous "snowballs" and peripheral retinal vasculitis with or without anterior chamber inflammation, and cystoid macular oedema. Significant chorioretinal inflammation was absent in all cases. After agreeing to participate, patients underwent a detailed interview, quality of life assessment, visual function evaluation and finally clinical examination, in that order. This study was approved by the hospital ethics committee and informed consent was obtained from all patients.

\section{Quality of life assessment}

HR-QOL was evaluated using the UK standard version of the SF-36, which consists of 36 items grouped into eight scales to measure health, including physical functioning, social functioning, role limitations due to physical problems, role limitations due to emotional problems, mental health, energy/vitality, bodily pain, and general health perception. ${ }^{8}$ The SF-36 subscale scores range from $0 \%$ to $100 \%$, with higher scores indicating better health. General population data for the UK version of the SF-36, which were used for comparison with the intermediate uveitis patients, were derived from the Oxford Healthy Life Survey in which HRQOL was evaluated in 13042 randomly selected subjects of working age. ${ }^{9}$ VR-QOL was measured using the VCMl, a 10 item questionnaire that provides a subjective measure of concern regarding vision with scores ranging from 0.0 (best score) to 5.0 (worst score) with 50 intervals. ${ }^{10}$ The questionnaires were self administered in approximately 10 minutes. The validity and reliability of the VCMI and the SF-36

Abbreviations: $\mathrm{BIO}$ score, binocular indirect ophthalmoscopy score $\mathrm{CS}$, contrast sensitivity; $\mathrm{CV}$, colour vision; $\mathrm{HR}-\mathrm{QOL}$, health related quality of life; IU, intermediate uveitis; MCS, mental component summary score; PCS, physical component summary score; SF-36 questionnaire, short form 36 questionnaire; VA, visual acuity; VF, visual field; VR-QOL, vision related quality of life 


\begin{tabular}{|lc|}
\hline Table 1 Clinical and demographic characteristics \\
\hline Variable & \\
\hline Mean age (years) (SD) & $41.1(14.3)$ \\
Sex (\%) & $25(59.5)$ \\
Female & $17(40.5)$ \\
Male & $4.7(3.5)$ \\
Mean duration of uveitis (years) (SD) & \\
Current treatment (\%) & $6(14.3)$ \\
None & $9(21.4)$ \\
Topical corticosteroids alone & $3(7.1)$ \\
Oral prednisolone alone & $23(54.8)$ \\
Oral prednisolone plus immunosuppressant(s)* & $1(2.4)$ \\
Immunosuppressant alone & \\
Laterality (\%) & $12(28.6)$ \\
Unilateral & $30(71.4)$ \\
Bilateral & $33(78.6)$ \\
Associated systemic disease (\%) & $6(14.3)$ \\
None & $3(7.1)$ \\
Sarcoidosis & \\
Multiple sclerosis & $24(57.1)$ \\
Medical co-morbidities (\%) & $7(16.7)$ \\
None & $2(4.8)$ \\
Hypertension & $2(4.8)$ \\
Ischaemic heart disease & $1(2.4)$ \\
Asthma & $1(2.4)$ \\
Hypothyroidism & $1(2.4)$ \\
Diabetes mellitus & \\
Rheumatoid arthritis & \\
\hline *Immunosuppressants included ciclosporin A, tacrolimus, mycophenolate \\
mofetil, azathioprine, and methotrexate. \\
\hline
\end{tabular}

as tools for measuring VR-QOL and HR-QOL in a variety of diseases have been well established. ${ }^{71}{ }^{12}$

\section{Visual function assessment}

Visual acuity (VA), contrast sensitivity (CS), colour vision $(\mathrm{CV})$, and visual field (VF) were assessed wearing usual distance spectacle correction, if any, first monocularly then binocularly. Best corrected $\log$ MAR VA scored for individual letters was measured at 4 metres with the ETDRS chart mounted on the Lighthouse Chart Illumination Unit (USA). Pelli-Robson CS was measured as recommended by the manufacturer and under controlled illumination; the screen luminance was $85-120 \mathrm{~cd} / \mathrm{m}^{2} .{ }^{13} \mathrm{CS}$ was scored letter by letter as this method gives greatest reliability. ${ }^{14}$ The Esterman test was used to record monocular and binocular VF on the Humphrey field analyser II perimeter. ${ }^{15}$ The VF was scored using the Esterman efficiency score, which is the percentage of test points correctly seen by the patient. CV was measured using the Farnsworth-Munsell 100 hue test under standard controlled lighting conditions, as described by the manufacturers. Published normal ranges for VA, CS, and CV were used for comparison with the intermediate uveitis patients to qualify the extent of their visual impairment. ${ }^{1416}$ An

Table 2 Frequency of sight threatening clinical features

\begin{tabular}{lc}
\hline Variable & $\begin{array}{l}\text { No }(\%) \text { of eyes } \\
(\mathbf{n}=72)\end{array}$ \\
\hline Macular involvement* & $51(70.8)$ \\
Optic nerve involvement† & $8(11.1)$ \\
BIO score $>1$ & $12(16.7)$ \\
Glaucoma & $1(1.4)$ \\
Cataract¥ & $13(18.1)$ \\
\hline
\end{tabular}

*Includes eyes with epiretinal membranes, retinal vasculitis affecting the posterior pole (defined on fundus fluorescein angiography), and those previously/currently affected by cystoid macular oedema.

tIncludes six eyes with previous optic disc swelling and two eyes with optic neuritis secondary to multiple sclerosis.

fDefined as greater than LOCS III grade 1 .

$\mathrm{BIO}$ score = binocular indirect ophthalmoscopy score.
Esterman efficiency score of less than 100 was considered abnormal.

\section{Clinical examination}

In each case, the ocular examination was performed by the same observer (EHH), who was masked to the outcome of the quality of life and visual function assessments and had no knowledge of the patients' past history. The international uveitis scoring system was used to grade uveitis activity. ${ }^{1}$ To facilitate comparisons, uveitis was graded as "inactive" if the anterior chamber was $1+$ or less and the binocular indirect ophthalmoscopy score (BIO) score was 0 , or "active" if more significant inflammation was present. Lens opacities were graded using the LOCS III method. ${ }^{18}$

\section{Data analysis}

To facilitate comparison with age and sex matched general population norms, the SF-36 subscale scores are presented as $\mathrm{T}$ scores, which were calculated using means and standard deviations derived from the age and sex matched UK normative data set. ${ }^{8}$ For $\mathrm{T}$ scores, the normative data set has a mean of 50 and a standard deviation of 10 so that patient scores greater than 50 represent better than average quality of life and those below 50 represent worse than average quality of life. The eight domains of the SF-36 were also summarised into the physical component summary score (PCS, derived from the physical functioning, role physical, bodily pain, and general health perception subscales) and the mental component summary score (MCS, derived from the energy/vitality, social functioning, role emotional, and mental health subscales) to reduce the number of SF-36 variables for statistical analysis. ${ }^{19}$

Statistical analyses were performed using Prism version 3.02 (GraphPad, San Diego, CA, USA) and SPSS version 10.0 for Windows. All tests were two tailed and statistical significance attributed when $\mathrm{p}<0.05$. Patient and normative data were compared using independent $t$ tests. The associations between visual function tests and quality of life scores were examined using Spearman correlation analysis. Logistic regression analysis was used to determine whether the following clinical and demographic variables were predictive of a VCMl score of more than 2.0: VA, CS, CV, VF, age, sex, medical co-morbidities, and uveitis duration, activity, laterality, and treatment (systemic immunosuppression or not). Systemic co-morbidities were recorded as present or absent, giving equal weight to all systemic conditions. Stepwise multiple linear regression analysis was used to determine the variance of the physical and mental component summary scores resulting from these same clinical and demographic variables.

\section{RESULTS}

\section{Clinical and demographic characteristics}

The clinical and demographic characteristics of the group are described in table 1 . An unusually high percentage of patients had unilateral disease (28.6\%). At the time of evaluation 27 (64.3\%) patients were taking systemic corticosteroids and/or immunosuppressive agents and, in the past, 33 (78.6\%) had required systemic therapy at some time to control their intraocular inflammation. Uveitis was inactive (anterior chamber cells +1 or less and BIO score zero in the absence of other signs of posterior segment activity) in 29 (69\%) patients. Clinical signs of activity that were present were cystoid macular oedema in $11(15.3 \%)$ eyes and BIO score of 2 or more in $12(16.7 \%)$ eyes. Table 2 indicates the frequency of sight threatening clinical features and the probable causes of visual impairment. Two patients had snow banking suggesting pars planitis. None of the patients had any ocular pathology unrelated to uveitis. 


\begin{tabular}{|c|c|c|c|c|c|}
\hline Visual function test & Minimum & Maximum & Median & Interquartile range & $\begin{array}{l}\text { Correlation } \\
\text { with VCM1 } r \text { (p } \\
\text { value) }\end{array}$ \\
\hline \multicolumn{6}{|c|}{ LogMAR VA (Snellen equivalent)) } \\
\hline Better eye & $-0.2(20 / 12.5)$ & $0.64(20 / 80)$ & $0.01(20 / 20)$ & $-0.10-0.10(20 / 15-20 / 25)$ & $0.03(0.85)$ \\
\hline Worse eye & $-0.1(20 / 15)$ & NPL & $0.19(20 / 32)$ & $0.03-0.43(20 / 20-20 / 50)$ & $0.33(0.035)$ \\
\hline Binocular & $-0.26(20 / 10)$ & $0.66(20 / 100)$ & $0.02(20 / 20)$ & $-0.10-0.12(20 / 15-20 / 25)$ & $0.07(0.68)$ \\
\hline \multicolumn{6}{|l|}{ CS (log units) } \\
\hline Better eye & 0.95 & 1.80 & 1.60 & $1.40-1.65$ & $0.01(0.96)$ \\
\hline Worse eye & 0.00 & 1.95 & 1.50 & $1.26-1.65$ & $-0.27(0.08)$ \\
\hline Binocular & 0.95 & 1.95 & 1.65 & $1.56-1.88$ & $-0.12(0.47)$ \\
\hline \multicolumn{6}{|l|}{ CV (total error score) } \\
\hline Better eye & 15 & 599 & 88 & $63-157$ & $-0.08(0.60)$ \\
\hline Worse eye & 18 & u & 82 & $58-136$ & $0.14(0.39)$ \\
\hline Binocular & 17 & 84 & 74 & $47-130$ & $0.05(0.73)$ \\
\hline \multicolumn{6}{|c|}{ VF (\% test points seen) } \\
\hline Better eye & 76 & 100 & 98 & $94-100$ & $-0.24(0.12)$ \\
\hline Worse eye & 0 & 100 & 97 & $88-99$ & $-0.34(0.026)$ \\
\hline Binocular & 42 & 100 & 98 & $94-100$ & $-0.35(0.02)$ \\
\hline
\end{tabular}

\section{Effect of intermediate uveitis on visual function}

Table 3 describes the VA, CS, CV, and VF of this intermediate uveitis cohort. VA was $0.3 \log$ MAR (equivalent to 20/40) or better in $77.8 \%$ of affected eyes. Three $(7.1 \%)$ patients had unilateral legal blindness, as defined in the United States as a VA of less than 1.0 logMAR (equivalent to 20/200) in the worse eye. ${ }^{20}$ Nine $(21.4 \%)$ patients had unilateral visual impairment, defined as a $\log M A R$ equal to or less than 0.5 (equivalent to 20/63) in their worse eye. ${ }^{21}$ VA, CS, CV, and VF were worse than published normal standards for this age group in $33(45.8 \%), 33(45.8 \%), 23(31.9 \%)$, and $54(75.0 \%)$ affected eyes $(\mathrm{n}=72)$, respectively. ${ }^{14}{ }^{16}{ }^{17}$ As shown in table 4 , significant correlations were found between all of the visual function tests. The closest association was found for VA and CS $(R=-0.69, p<0.0001)$. Longer duration of disease was associated with worse VA (Spearman $r=0.512, \mathrm{p}<0.0001$ ) but not worse VR-QOL.

\section{Vision related quality of life}

The median (interquartile range) VCMl score was 0.8 (0.51.4). As shown in figure $1,9.5 \%$ of patients had a VCMl score of more than 2.0, which represents "more than a little" concern about vision. ${ }^{11}$ The VCMl correlated with worse eye VA, worse eye VF, and binocular VF but did not correlate with any of the better eye visual function tests, as shown in table 3 . In the logistic regression analysis, worse eye VA $(p=0.045)$ and worse eye CS $(p=0.042)$ were predictive of a VCMl score of more than 2.0 independently of age, sex, uveitis duration, laterality and activity, systemic uveitis therapy, and medical co-morbidity. The other visual function tests, including the better eye and binocular results, were not associated with the VCMI score in the regression analysis.

\section{Health related quality of life}

The SF-36 subscales social functioning, general health perception, and pain were significantly worse for the

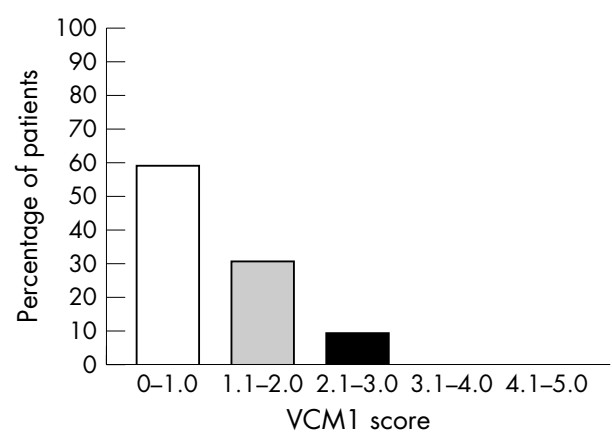

Figure 1 Distribution of VCM1 scores.

intermediate uveitis patients than the general population in the United Kingdom when matched for age and sex, as shown in table $5(\mathrm{p}=0.0002, \mathrm{p}<0.0001$ and $\mathrm{p}=0.01$, respectively). ${ }^{9}$ The other SF-36 subscales were similar to the general population. In the multivariate regression analysis, the PCS and MCS were not associated with age, sex, uveitis duration, treatment, laterality or activity, the presence of systemic co-morbidity, or any of the measures of visual function. However, the VCMI was predictive of the MCS score after adjusting for the above variables $\left(R^{2}=0.26\right.$, $\mathrm{p}=0.001)$. The VCMl did not have significant predictive value with regard to the PCS score.

Table 6 indicates the correlations between the SF-36 subscales and the visual function tests and VCMI. Significant correlations were found between the VCMI and all of the SF-36 scales $(r=-0.39$ to $-0.68, \mathrm{p}<0.05$ for all). Correlations between the visual function tests and the SF-36 subscales were mostly not significant and as with the VCMl, better eye visual function had no correlation with the SF-36 subscale scores. Notably, however, worse binocular VF was

Table 4 Visual function test correlation matrix ( $n=72$ eyes)

\begin{tabular}{llll}
\hline \multicolumn{1}{c}{ VA } & CS & CV & VF \\
\hline VA & $-0.69(<0.0001)$ & $0.54(<0.0001)$ & $-0.53(<0.0001)$ \\
CS & & $-0.61(<0.0001)$ & $0.57(<0.0001)$ \\
CV & & $0.33(0.0046)$ \\
VF & & \\
\hline VA, visual acuity; CS, contrast sensitivity; CV, colour vision; VF, visual. field. Data presented are Spearman \\
correlation coefficients and p values (in parentheses).
\end{tabular}


Table 5 SF-36 subscale T scores for the intermediate uveitis patients and the age and sex matched general population in the United Kingdom

\begin{tabular}{llll}
\hline & $\begin{array}{l}\text { General population } \\
\text { score } \\
\text { Mean (SD) }\end{array}$ & $\begin{array}{l}\text { Intermediate uveitis } \\
\text { score } \\
\text { Mean (SD) } \\
\text { range 0-100 }\end{array}$ & p Value \\
SF-36 subscale & $50(10)$ & $49.3(13.3)$ & 0.66 \\
\hline Physical functioning (PF) & $50(10)$ & $47.5(13.3)$ & 0.12 \\
Role physical (RP) & $50(10)$ & $48.6(11.7)$ & 0.37 \\
Role emotional (RE) & $50(10)$ & $44.0(14.8)$ & 0.0002 \\
Social functioning (SF) & $50(10)$ & $49.2(11.6)$ & 0.62 \\
Mental health (MH) & $50(10)$ & $47.1(12.9)$ & 0.07 \\
Energy and vitality (EV) & $50(10)$ & $45.8(11.6)$ & 0.01 \\
Pain (P) & $50(10)$ & $42.6(12.3)$ & $<0.0001$ \\
General health perception (GHP) & $50(10)$ & $47.7(8.4)$ & 0.15 \\
Physical component summary (PCS) & $50(10)$ & $48.1(8.3)$ & 0.23 \\
Mental component summary (MCS) & & & \\
\hline Statistically significant results are in bold. & & &
\end{tabular}

associated with worse SF-36 scores across all subscales except role emotional, general health perception, and the physical component summary score. Patients who expressed "more than a little" concern over their vision (VCMl score >2.0) had significantly worse PCS and MCS scores than patients who had expressed little or no concern over their vision $(\mathrm{VCMl}<2.1)$, as shown in figure 2 .

\section{DISCUSSION}

This study explored the impact of intermediate uveitis on visual performance and self reported vision and health related quality of life. Despite the severe disease in this cohort, reflected by the need for systemic immunotherapy in the past in almost $80 \%$ of the patients, over half of the eyes maintained visual acuity and contrast sensitivity of 0.1 $\log$ MAR (equivalent to 20/25) and $1.55 \log$ units or better, the lower limits of normal. ${ }^{14}{ }^{16}$ Furthermore, only two $(4.7 \%)$ patients had a VA of worse than 0.3 logMAR (equivalent to 20/40) in their better eye, the minimum required for driving in the United Kingdom, and none of the patients was legally blind. These results compare with those of Rothova et al, who described the prevalence and severity of visual impairment in a cohort of 78 patients with intermediate uveitis as part of a large retrospective analysis of visual loss in uveitis. ${ }^{21}$

This is the first study to our knowledge to describe colour vision and visual field performance in intermediate uveitis. Colour vision was the least affected of the four measures of visual function; it was recorded as normal in over two thirds of uveitic eyes and correlated poorly with the patients' perceptions of their visual performance as measured with the VCM1. Visual field loss was recorded in three quarters of uveitic eyes by the monocular Esterman visual field test. As previously reported, the most common cause of visual impairment was macular involvement, usually manifested as cystoid macular oedema or as an epiretinal membrane. ${ }^{21}$
Only $9.5 \%$ of the patients reported "more than a little" concern over their vision, suggesting that vision is well preserved in the vast majority of patients with intermediate uveitis. Schiffman et al described vision and health related quality of life in 76 patients with various types of uveitis and concluded that uveitis has a "more pervasive impact on VRQOL than does age related macular degeneration." ${ }^{\prime 4}$ Only $22 \%$ of their cohort had intermediate uveitis and it is likely that the greater visual impairment recorded in their series compared with the present study resulted from the greater proportion of patients with posterior and panuveitis.

Worse eye VA and CS were predictive of the VCMI score after adjusting for other clinical and demographic variables and significant correlations were found between the VCMI and the worse eye VA and VF but not the better eye results. This contrasts with the usual finding that VR-QOL is more closely associated with the better eye VA, as reported for cataract, age related macular degeneration, and patients awaiting a corneal graft. ${ }^{22-24}$ Gardiner et al used the VCMI to evaluate visual performance in a group of 132 patients with various types of uveitis and found that worse eye, better eye and binocular high contrast VA correlated with VR-QOL. ${ }^{5}$

Although the mean HR-QOL scores of the intermediate uveitis cohort were worse than the age and sex matched population average for all eight domains of the SF-36, this difference only reached significance for the subscales social functioning, general health perception, and pain. In the regression analysis, none of the variance of the physical and mental component summary scores could be attributed to any of the patients' clinical or demographic characteristics recorded or the clinical measures of visual function. The impact of subjective visual functioning on HR-QOL in intermediate uveitis was confirmed by the significantly worse PCS and MCS scores in patients with a VCMl score greater than 2.0. Strong correlations between the SF-36 subscales
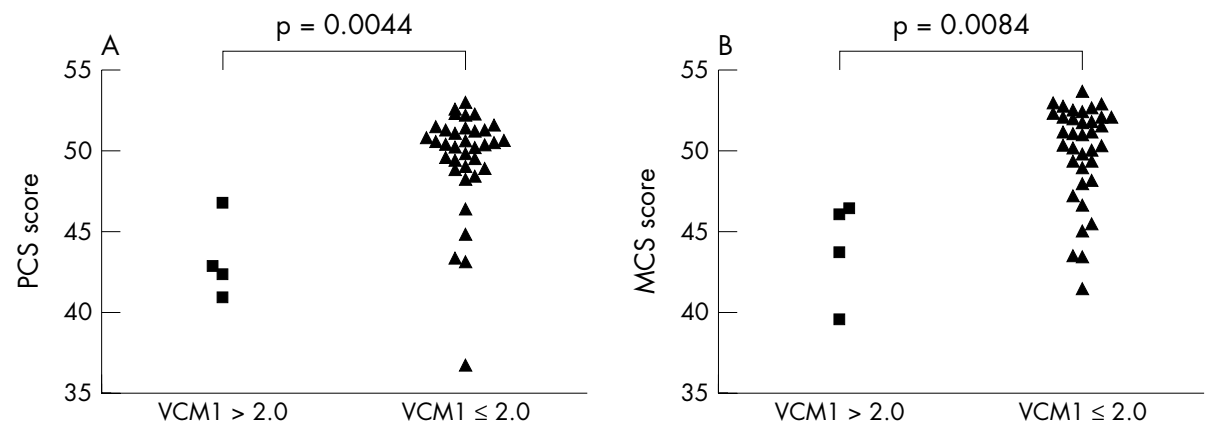

Figure 2 PCS scores (A) and MCS scores (B) for patients with (VCMI $>2.0)$ and without (VCMI $\leqslant 2.0)$ "more than a little" concern over their vision. 
Table 6 Correlation between the VCMI visual function tests, and SF-36 subscale measures

\begin{tabular}{|c|c|c|c|c|c|c|c|c|c|c|}
\hline & PF & RP & RE & SF & MH & EV & $\mathbf{P}$ & GHP & PCS & MCS \\
\hline VCMI & $\begin{array}{l}-0.39 \\
(0.01)\end{array}$ & $\begin{array}{l}-0.54 \\
(<0.0001)\end{array}$ & $\begin{array}{l}-0.44 \\
(0.004)\end{array}$ & $\begin{array}{l}-0.40 \\
(0.026)\end{array}$ & $\begin{array}{l}-0.53 \\
(<0.0001)\end{array}$ & $\begin{array}{l}-0.51 \\
(0.001)\end{array}$ & $\begin{array}{l}-0.51 \\
(0.001)\end{array}$ & $\begin{array}{l}-0.49 \\
(0.001)\end{array}$ & $\begin{array}{l}-0.68 \\
(<0.0001)\end{array}$ & $\begin{array}{l}-0.57 \\
(<0.0001)\end{array}$ \\
\hline \multicolumn{11}{|c|}{ 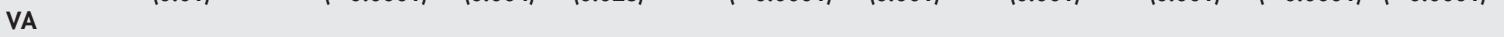 } \\
\hline Better eye & -0.23 & -0.05 & 0.01 & 0.11 & 0.14 & -0.16 & -0.09 & -0.22 & -0.33 & -0.02 \\
\hline Worse eye & -0.21 & -0.19 & -0.14 & -0.07 & -0.06 & -0.19 & $-0.34(0.03)$ & -0.29 & -0.21 & -0.14 \\
\hline $\begin{array}{l}\text { Binocular } \\
\text { CS }\end{array}$ & \multicolumn{9}{|c|}{ CS } & 0.01 \\
\hline Better eye & 0.04 & -0.14 & -0.06 & -0.12 & -0.19 & -0.06 & -0.11 & 0.00 & -0.12 & -0.09 \\
\hline Worse eye & 0.15 & 0.13 & 0.18 & 0.15 & 0.10 & $0.33(0.035)$ & -0.12 & 0.23 & 0.14 & 0.24 \\
\hline $\begin{array}{l}\text { Binocular } \\
\text { CV }\end{array}$ & \multicolumn{6}{|c|}{ CV } & 0.12 & 0.12 & & 0.05 \\
\hline Befter eye & -0.17 & 0.16 & -0.02 & -0.04 & 0.18 & -0.09 & 0.01 & -0.20 & -0.04 & -0.04 \\
\hline Worse eye & -0.16 & 0.12 & -0.04 & -0.06 & 0.12 & -0.17 & -0.15 & -0.14 & -0.13 & -0.15 \\
\hline Binocular & -0.14 & 0.27 & -0.01 & -0.01 & 0.04 & -0.14 & -0.12 & -0.14 & -0.05 & -0.09 \\
\hline VF & & & & & & & & & & \\
\hline Better eye & 0.27 & 0.17 & 0.19 & 0.04 & 0.04 & 0.15 & 0.13 & 0.10 & -0.02 & 0.16 \\
\hline Worse eye & 0.26 & $0.44(0.004)$ & 0.23 & 0.17 & 0.23 & $0.31(0.048)$ & 0.25 & 0.14 & 0.14 & 0.30 \\
\hline Binocular & $0.38(0.014)$ & $0.43(0.004)$ & 0.28 & $0.42(0.005)$ & $0.35(0.024)$ & $0.44(0.004)$ & $0.35(0.023)$ & 0.21 & 0.20 & $0.44(0.003)$ \\
\hline
\end{tabular}

Data presented are Spearman correlation coefficients and significant $\mathrm{p}$ values (in parentheses). Significant results are in bold. See table 5 for abbreviations.

and the VCMl suggest that vision is the key factor that influences global quality of life in intermediate uveitis.

In conclusion, this study demonstrates that visual function and general health are well maintained in the majority of patients with intermediate uveitis and highlights the impact of impaired vision related quality of life on general health related quality of life in this form of uveitis.

\section{Authors' affiliations}

C C Murphy, E H Hughes, A D Dick, Division of Ophthalmology, University of Bristol, Bristol, UK

N A Frost, Department of Ophthalmology, Torbay General Hospital, Torquay, UK

Grant support: This study was supported by Fujisawa, UK and The National Eye Research Centre, UK.

Competing interests: none declared

\section{REFERENCES}

1 Benezra D, Forrester JV, Nussenblatt RB, et al. Uveitis scoring system. Berlin: Springer-Verlag, 1991:8.

2 Legro MW. Quality of life and cataracts: a review of patient-centered studies of cataract surgery outcomes. Ophthalmic Surg 1991;22:431-43.

3 Parrish RK, Gedde SJ, Scott IU, et al. Visual function and quality of life among patients with glaucoma. Arch Ophthalmol 1997;115:1447-55.

4 Schiffman RM, Jacobson G, Whitcup SM. Visual functioning and general health status in patients with uveitis. Arch Ophthalmol 2001;119:841-9.

5 Gardiner AM, Armstrong RA, Dunne MC, et al. Correlation between visual function and visual ability in patients with uveitis. $\mathrm{Br} J$ Ophthalmol 2002;86:993-6.

6 Mackenzie PJ, Chang TS, Scott IU, et al. Assessment of vision-related function in patients with age-related macular degeneration Ophthalmology 2002; 109:720-9

7 Ware JE Jr. SF-36 health survey update. Spine 2000;25:3130-9.

8 Jenkinson C, Layte R, Wright L, et al. The UK-36: an analysis and interpretation manual. Oxford: Joshua Horgan print partnership, 1996.
9 Jenkinson C, Coulter A, Wright L. Short form 36 (SF-36) health survey questionnaire: normative data for adults of working age. BMJ 1993:306: 1437-40

10 Frost NA, Sparrow JM, Durant JS, et al. Development of a questionnaire for measurement of vision-related quality of life. Ophthalmic Epidemiol 1998;5:185-210.

11 Frost A, Eachus J, Sparrow J, et al. Vision-related quality of life impairment in an elderly UK population: associations with age, sex, social class and material deprivation. Eye 2001;15:739-44.

12 Frost NA, Sparrow JM, Hopper CD, et al. Reliability of the VCMI questionnaire when administered by post and by telephone. Ophthalmic Epidemiol 2001;8:1-11.

13 Pelli DG, Robson JG, Wilkins AJ. The design of a new letter chart for measuring contrast sensitivity. Clin Vis Sci 1988;2:187-99.

14 Elliott DB, Bullimore MA, Bailey IA. Improving the reiliability of the PelliRobson contrast sensitivity test. Clin Vis Sci 1991;6:471-5.

15 Esterman B. Functional scoring of the binocular field. Ophthalmology 1982:89:1226-34

16 Elliott DB, Hurst MA. Simple clinical techniques to evaluate visual function in patients with early cataract Optom Vis Sci 1990;67:822-5.

17 Verriest G, Van Laethem J, Uviils A. A new assessment of the normal ranges of the Farnsworth-Munsell 100-hue test scores. Am J Ophthalmol 1982;93:635-42.

18 Chylack LT Jr. Wolfe JK, Singer DM, et al. The Lens Opacities Classification System III. The Longitudinal Study of Cataract Study Group. Arch Ophthalmol 1993;111:831-6.

19 Jenkinson C. The SF-36 physical and mental health summary measures: an example of how to interpret scores. $J$ Health Serv Res Policy 1998;3:92-6.

20 Kraut KA, McCabe CP. The problem of low vision. Definition and common problems. In, Albert DM, Jakobiec FA, eds. Principles and practice of ophthalmology Philadelphia, WB Saunders, 1994:3664-6.

21 Rothova A, Suttorp-van Schulten MS, Frits Treffers W, et al. Causes and frequency of blindness in patients with intraocular inflammatory disease. Br J Ophthalmol 1996:80:332-6.

22 Steinberg EP, Tielsch JM, Schein O, et al. An index of functional impairment in patients with cataract. Arch Ophthalmol 1994;112:630-8.

23 Miskala PH, Bressler NM, Meinert CL. Relative contributions of reduced vision and general health to NEI-VFQ scores in patients with neovascular agerelated macular degeneration. Arch Ophthalmol 2004;122:758-66.

24 Boisjoly H, Gresset J, Fontaine N, et al. The VF-14 index of functional visual impairment in candidates for a corneal graft. Am J Ophthalmol $1999 ; 128: 38-44$ 